K. Nishioka

Nagoya Math. J.

Vol. 113 (1989), 173-179

\title{
DIFFERENTIAL ALGEBRAIC FUNCTION FIELDS DEPENDING RATIONALLY ON ARBITRARY CONSTANTS
}

\author{
KEIJI NISHIOKA
}

\section{§1. Introduction}

The general solution of an algebraic differential equation depends on the initial conditions, though it is in general too difficult to make explicit the shape of the relationship. Painlevé studied in [8] algebraic differential equations of second order with the general solutions depending rationally on the initial conditions and the solvability of such equations. Giving the precise definition of the notion "rational dependence on the initial conditions", Umemura [10] revived and generalized rigorously the discussion of Painlevé in the language of modern algebraic geometry. The theorem of Umemura is as follows; Let $K$ be a differential field extension of complex number field $C$ generated by a finite number of meromorphic functions on some domain in $C$. Let $y$ be the general solution of a given algebraic differential equation over $K$. Suppose that $y$ depends rationally on the initial conditions. Then it is contained in the terminal $K_{m}$ of a finite chain of differential field extensions: $K=K_{0} \subset K_{1} \subset \cdots \subset K_{m}$ such that each $K_{i}$ is strongly normal over $K_{i-1}$.

In [5] the author defined the following: Let $K$ be an ordinary differential field of characteristic zero. A differential field extension $L$ of $K$ is said to depend rationally on arbitrary constants if there exists a differential field extension $M$ of $K$ such that $L$ and $M$ are free over $K$ and $L M=M C_{L M}$, where $C_{L M}$ denotes the field of constants of $L M$. Two notions "the rational dependence on the initial conditions" and "the rational dependence on arbitrary constants" are equivalent. The later originates directly in the author's [4] (see also Matsuda [3]). The objective of this paper is to prove the following:

Received August 25, 1986.

Revised January 6, 1988. 
Theorem. Let $K$ be an algebraically closed ordinary differential field of characteristic zero. Let $R$ be a differential field extension of $K$ generated by a single element which is differentially algebraic over $K$. Then the following are equivalent:

(i) $C_{R}=C_{K}$ and $R$ depends rationally on arbitrary constants;

(ii) there exists a strongly normal extension of $K$ which contains $R$.

This tells us that the length of the chain needed in the conclusion of Umemura's theorem is at most 2. It will be worthy to remark a wellknown fact that if $K$ contains nonconstants any differential field extension of $K$ that is a finitely generated field extension of $K$ contains an element $y$ with $R=K\langle y\rangle$ (for instance see Ritt [9]).

The proof of Theorem will be divided into 4 steps. We utilize some basic facts in Kolchin [2] without warning and discuss entirely from differential-algebraic viewpoint.

\section{§2. The proof of Theorem}

The deduction of (ii) from (i) is a straightforward result from Lemma 1 of [7] (see below). So it is sufficient to prove (i) under the condition (ii). Let $U$ be a universal differential field extension of $K$. For any intermediate differential field $L$ between $K$ and $U$ we denote by $C_{L}$ the field of constants of $L$. Here we recall the definition of strongly normal extensions: A finitely generated differential field extension $N$ of $K$ is called a strongly normal extension of $K$ if $C_{N}=C_{K}$ and there exists a differential subfield $M$ of $U$ containing $K$ such that $M$ is differentially isomorphic to $N$ over $K, M$ and $N$ are linearly disjoint over $K$ and $M N=M C_{M N}$ (cf. Proposition 1 of [1]). We extract Lemma 1 of [7] for reader's convenience:

LEMma. Let $L$ and $M$ be two intermediate differential fields between $K$ and $U$. Suppose $M$ is a finitely generated differential field extension of $L$ which is contained in $L C_{U}$. Then $M=L C_{M}$.

Now let us return to the proof of our theorem. By assumption of Theorem there is an element $y$ of $R$ with $R=K\langle y\rangle$ and tr.deg $R / K$ is finite. Assume (i). Then there exists a finitely generated differential field extension $E$ of $K$ such that $E$ and $R$ are linearly disjoint over $K$ and $E R=E C_{E R}$. We have generators $e=\left(e_{i}\right)_{1 \leqq i \leqq p}$ of $E$ over $K: E=K\langle e\rangle$. 
Step 1. There exists a finitely generated differential field extension $E_{1}$ of $K$ such that $E_{1}$ and $R$ are linearly disjoint over $K, C_{E_{1}}=C_{K}$ and $E_{1} R=E_{1} C_{E_{1} R}$.

Proof. Since $y$ is in $E R=E C_{E R}=K\langle e\rangle C_{E R}$, we have the representation:

$$
y=\sum a_{j} x_{j} / \sum b_{j} x_{j}, \quad 1 \leqq j \leqq q
$$

where the $a$ 's and $b$ 's are elements of $C_{E R}$, the $x$ 's are elements of $K\{e\}$ which are linearly independent over $C_{E R}$ and $b_{i}$ does not vanish for some $i$. Since $a_{j}$ and $b_{j}$ lie in $E R$, we have the representations:

$$
\begin{aligned}
& a_{j}=\sum a_{j h} y_{h} / \sum b_{j h} y_{h}, \quad 1 \leqq h \leqq r \\
& b_{j}=\sum c_{j h} y_{h} / \sum d_{j h} y_{h}, \quad 1 \leqq h \leqq r
\end{aligned}
$$

where the $y$ 's are elements of $R$ which are linearly independent over $K$ and $a_{j h}, b_{j h}, c_{j h}, d_{j h}$ are elements of $K\{e\}$ such that for any $j$ there are $j_{1}$ and $j_{2}$ with $b_{j j_{1}} d_{j j_{2}} \neq 0$ and $c_{i h} \neq 0$ for some $h$. By $w$ we denote the wronskian of $\left(x_{j}\right)$. Then the linear independence of $\left(x_{j}\right)$ over constants implies $w \neq 0$. Note that $w$ is an element of $K\{e\}$. By Kolchin [2] there exists a differential homomorphism $\phi$ of $K\{e\}$ to $U$ over $K$ such that $\phi\left(b_{j j_{1}} d_{j j_{2}}\right) \neq 0$ for any $j, \phi\left(w c_{i n}\right) \neq 0$ and $C_{K\langle\phi e\rangle}=C_{K}$. It is readily seen from the universality of $U$ that there exists a differential field extension $E_{1}$ of $K$ such that $E_{1}$ is differentially isomorphic to $K\langle\phi e\rangle$ and $E_{1}$ and $R$ are linearly disjoint over $K$. Then $\phi$ can be extended to a differential homomorphism of $R\{e\}$ to $E_{1} R$ over $R$. We denote this by the same symbol $\phi$. Since $\phi w \neq 0$ we see $\left(\phi x_{j}\right)$ are linearly independent over constants. Noting also $\phi b_{i}$ is defined and nonzero because $\left(y_{h}\right)$ are linearly independent over $E_{1}$ and $\phi c_{i n} \neq 0$ we have

$$
\phi \sum b_{j} x_{j}=\sum \phi b_{j} \phi x_{j} \neq 0 \text {. }
$$

And

$$
y=\phi y=\sum \phi a_{j} \phi x_{j} / \sum \phi b_{j} \phi x_{j}
$$

is contained in $E_{1} C_{E_{1} R}$ since $\phi a_{j}$ and $\phi b_{j}$ belong to $C_{E_{1} R}$. Thus we conclude $R$ is contained in $E_{1} C_{E_{1} R}$ and so $E_{1} R=E_{1} C_{E_{1} R}$.

Step 2. There exists a finitely generated differential field extension $E_{2}$ of $K$ such that $E_{2}$ and $R$ are linearly disjoint over $K, C_{E_{2}}=C_{K}, E_{2} R$ $=E_{2} C_{E_{2} R}$ and $E_{2}$ contains an element $z$ which is a generic differential specialization of $y$ over $K$. 
Proof. Take a generic differential specialization $z$ of $y$ over $K$ with the property that $K\langle z\rangle$ and $E_{1} R$ are linearly disjoint over $K$. This is possible according to the universality of $U$. Let $L$ denote the algebraic closure of $K\langle z\rangle$ in $U$. Then $L E_{1}$ and $L R$ are linearly disjoint over $L$. In fact $L$ and $E_{1} R$ are linearly disjoint over $K$ and so $E_{1} R$ and $E_{1} L$ are linearly disjoint over $E_{1}$. Hence $R$ and $L E_{1}$ are linearly disjoint over $K$. This implies our assertion. Clearly $L E_{1} R=L E_{1} C_{E_{1} R}$. Similarly to Step 1 we can find a finitely generated differential field extension $F$ of $L$ such that $L R$ and $F$ are linearly disjoint over $L, C_{F}=C_{L}=C_{K}$ and $F R=$ $F C_{F R}$. Take a differential subfield $F_{1}$ of $F$ which is a finitely generated differential field extension of $K$ and satisfies $R=F_{1} C_{F R}$. Then $F_{1}$ and $R$ are linearly disjoint over $K, C_{F_{1}}=C_{K}$ and $F_{1} R=F_{1} C_{F_{1} R}$ by virtue of Lemma. As $E_{2}$ we may take $F_{1}\langle z\rangle$. The verification of required properties is easy.

By the finite generatedness of $E_{2}, C_{E_{2} R}$ is finitely generated over $C_{K}$ in the ordinary sense. Hence we have elements $u=\left(u_{j}\right)_{1 \leqq j \leqq s}$ with $C_{E_{2} R}=C_{K}(u)$ and the representation:

$$
y=f_{1}(u) / f(u),
$$

where $f_{1}$ and $f$ are in $E_{2}[u], f \neq 0$. We may write

$$
u_{j}=g_{j}(y) / g(y),
$$

where $g_{j}$ and $g$ are in $E_{2}\{y\}, g \neq 0$. By substitution we get

$$
g\left(f_{1}(u) / f(u)\right)=h(u) / f(u)^{d},
$$

where $h$ is in $E_{\varepsilon}[u]$ and $d$ is a natural number. Let $X$ be the set of all $c$ in $C_{K}^{p}$ that are specializations of $u$ over $C_{K}$ and $V$ be the set of all $c$ in $X$ witb $f(c) h(c) \neq 0$. Then $X$ is an irreducible affine variety, $V$ is open and dense in $X$. In fact noting $K$ is algebraically closed we see $C_{E_{2}}=C_{K}$ is also algebraically closed. Hence

$$
E_{2}[u]=E_{2} \otimes_{C_{K}} C_{K}[u] .
$$

From this we have a representation $f h=\sum a_{j} p_{j}$, where the $a_{j}$ are in $E_{2}$, linearly independent over $C_{K}$, the $p_{j}$ are in $C_{K}[u]$. Using this representation we find that $c$ is an element of $V$ is equivalent to that $f h(c) \neq 0$ and therefore that $p_{j}(c) \neq 0$ for some $j$. This shows $V$ is open in $X$. Denote by $E_{3}$ the differential field extension of $K$ generated with $z$ and all $y(c), c$ being in $V$. Then $E_{3}$ is a differential subfield of $E_{2}$ with the 
finite transcendence degree over $K$ since the generators are all differentially algebraic over $K$ and $E_{2}$ is a finitely generated differential field extension of $K$. The element $y(c)$ of $E_{3}$ is a differential specialization of $y$ over $K$ associated with the specialization $c$ of $u$ over $C_{K}$. Note that any element $c$ of $V$ is characterized by $f(c) \neq 0, g(y(c)) \neq 0$.

Step 3. $E_{3} R=E_{3} C_{E_{3} R}$.

Proof. The field $E_{2}(u)$, the quotient field of $E_{2} \otimes_{C_{K}} C_{K}[u]$, is the function field of the irreducible variety $X^{\prime}$ determined from $X$ by base extension $C_{K} \rightarrow E_{2}$. Let us show that $V$ is dense in $X^{\prime}$. Let $t$ be a regular function on $X^{\prime}$. Then we can express as $t=\sum a_{i} t_{i}, a_{i}$ in $E_{2}, t_{i}$ in $C_{K}[u]$, the $a_{i}$ being linearly independent over $C_{K}$. For any $c$ in $V$ we have $t(c)=\sum a_{i} t(c)$. If $t(c)=0$ then $t_{i}(c)=0$ for all $i$. Each $t_{i}$ would be identically 0 on $X$ and hence 0 on $X^{\prime}$. Thus $t=0$ on $X^{\prime}$. This shows $V$ is dense in $X^{\prime}$. Let $F$ be the algebraic closure of $E_{3}$ in $E_{2}$. We regard $E_{2}$ as a field with operators in the sense of [1], where as operators we take $\operatorname{Der}\left(E_{2} / E_{3}\right)$. Then $F$ is the field of constants of $E_{2}$. Since $E_{2}$ and $E_{3}(u)$ are linearly disjoint over $E_{3}$, we may set $D u_{i}=0$ for each $i$ and each derivative $D$ in $\operatorname{Der}\left(E_{2} / E_{3}\right)$, and $E_{2}(u)$ becomes a field extension of $E_{3}(u)$ in the sense of [1]. For any $D$ in $\operatorname{Der}\left(E_{2} / E_{3}\right)$ we have $D y(c)=0$ and

$$
f(c) D f_{1}(c)-f_{1}(c) D f(c)=0
$$

holds for each $c$ in $V$. From this and the fact $V$ is dense in $X^{\prime}$ it follows

$$
f D f_{1}-f_{1} D f=0
$$

holds in $E_{2}(u)$. Thus $D y=0$ and hence $y$ is in $F(u)$ since $F(u)$ is the field of constants of the field $E_{2}(u)$ with operators $\operatorname{Der}\left(E_{2} / E_{3}\right)$ according to Lemma 1 of [1]. Thus $F R$ is included in $F C_{E_{2} R}$ and by Lemma it follows $F R=F C_{F R}$. We assume $F$ is normal over $E_{3}$ by enlarging $F$ if necessary. We have $C_{F}=C_{K}$ recalling $C_{E_{2}}=C_{K}$. By defining $\tau u_{i}=u_{i}$ for each $i$ and any $\tau$ of $G\left(F / E_{3}\right)$, the Galois group of $F$ over $E_{3}$, we find $F(u)$ is normal over $E_{3}(u)$ and the Galois group is identified with $G\left(F / E_{3}\right)$. Now from $\tau y(c)=y(c) \in E_{3}$ it follows

$$
f(c) \tau f_{1}(c)-f_{1}(c) \tau f(c)=0
$$

holds for any $c$ in $V$, hence 


$$
f \tau f_{1}-f_{1} \tau f=0
$$

holds in $F(u)$ because $V$ is dense in $X^{\prime}$. This shows $\tau y=y$ and $y$ lies in $E_{3}(u)$.

By Step 3 we may assume from the first that $E$ has the finite transcendence degree over $K, C_{E}=C_{K}$ and $E$ has an element $z$ which is a generic differential specialization of $y$ over $K$. Take an $E_{4}$ among such $E$ 's with the least transcendence degree over $K$. Define $M$ from $E_{4}$ in the same manner as we defined $E_{3}$ from $E_{2}$. Then the degree of $E_{4}$ over $M$ is finite. We use the same notations such as $u, f_{1}, f, g_{j}, g$. Let $F$ be a normal algebraic extension of $M$ which contains $E_{4}$. As before we suppose $F(u)$ is normal over $E_{4}(u)$ and identify the Galois group $G\left(F(u) / E_{4}(u)\right)$ with $G\left(F / F_{4}\right)$. Then

$$
y=f_{1} / f=f_{1}^{*} / f^{*},
$$

where $f_{1}^{*}=f_{1} \prod_{\tau \neq 1} \tau f, f^{*}=\prod_{\tau} \tau f \in F[u], \tau \in G\left(F / E_{4}\right)$. Since $f^{*}$ is left invariant under any $\tau$ it is contained in $M(u)$ and therefore in $M[u]$. This is derived from the fact $F$ and $M(u)$ are linearly disjoint over $M$. By $M R=M C_{M R}=M(u)$ it follows $f_{1}^{*}=y f^{*}$ belongs to $M[u]$. Similarly we obtain the representation:

$$
u_{j}=g_{j} / g=g_{j}^{*} / g^{*},
$$

where $g_{j}^{*}=g_{j} \prod_{\tau \neq 1} \tau g, g^{*}=\prod_{\imath} \tau g \in M\{y\}, \quad \tau \in G\left(F / E_{4}\right)$. And from the fact $F$ and $M(u)=M\{y\}$ are linearly disjoint over $M$ it follows that $g^{*}$ and $g_{j}^{*}$ are contained in $M\{y\}$. By the definitions of $f^{*}$ and $g^{*}$ every element $c$ of $V$ is characterized by $f^{*}(c) \neq 0, g^{*}(y(c)) \neq 0$. Take a differential field extension $N$ of $K$ such that $N$ and $M$ are differentially isomorphic and linearly disjoint over $K$ and $N$ contains $R$.

Step 4. $N$ is a strongly normal extension of $K$.

Proof. There are a finite number of elements $\left(c_{h}\right)_{1 \leqq n \leqq m}$ of $V$ for which $M=K\left\langle z, z_{1}, \cdots, z_{m}\right\rangle, z_{h}=y\left(c_{h}\right)$. We can write $N=K\left\langle y, y_{1}, \cdots, y_{m}\right\rangle$, where each $y_{h}$ is a generic differential specialization of $z_{h}$ over $K$. Since $N$ and $M$ are linearly disjoint over $K$ we see $z_{h}$ is a differential specialization of $y_{h}$ over $M$ and $y_{h}$ is a differential specialization of $y$ over $M$. From $g^{*}\left(z_{h}\right) \neq 0$ we get $g^{*}\left(y_{h}\right) \neq 0$. Define $v_{h}=\left(v_{h \mathrm{j}}\right)$ by

$$
v_{h j}=g_{j}^{*}\left(y_{h}\right) / g *\left(y_{h}\right) \text {. }
$$


Then $\left(z_{h}, c_{h}\right)$ is a differential specialization of $\left(y_{h}, v_{h}\right)$ over $M$ and $\left(y_{h}, v_{h}\right)$ is a differential specialization of $(y, u)$ over $M$. All $v_{h}$ are elements of $C_{M\left\langle y_{h}\right\rangle}$. The equality $y f^{*}=f_{1}^{*}$ implies $y_{h} f^{*}\left(v_{h}\right)=f_{1}^{*}\left(v_{h}\right)$ and so $y_{h}$ is contained in $M\left(v_{h}\right)$. This derives $M N=M\left(u, v_{1}, \cdots, v_{m}\right)$ and completes the proof of Theorem.

\section{REFERENCES}

[1] A. Białynicki-Birula, On Galois theory of fields with operators, Amer. J. Math., 84 (1962), 89-109.

[2] E. R. Kolchin, Existence theorems connected with the Picard Vessiot theory of homogeneous linear ordinary differential equations, Bull. Amer. Math. Soc., 54 (1948), 927-932.

[ 3 ] - Differential Algebra and Algebraic Groups, Academic Press, New York, 1973.

[4] M. Matsuda, First order algebraic differential equations, A differential algebraic approach, Lecture Notes in Math., 804, Springer, Berlin, 1980.

[ 5 ] K. Nishioka, Algebraic differential equations of Clairaut type from the differentialalgebraic standpoint, J. Math. Soc. Japan, 31 (1979), 553-559.

[6] - A note on the transcendency of Painlevé's first transcendent, Nagoya Math. J., 109 (1988), 63-67.

[7] - General solutions depending algebraically on arbitrary constants, Nagoya Math. J., 113 (1989), 1-6.

[ 8 ] P. Painlevé, Leçon de Stockholm, OEuvres de P. Painlevé I, 199-818, Éditions du C.N.R.S., Paris, 1972.

[ 9 ] J. F. Ritt, Differential algebra, Amer. Math. Soc. Colloq. Publ. Vol. 33, New York, 1950.

[10] H. Umemura, Birational automorphism groups and differential equations, to appear in the Proc. Franco-Japanese colloquium on differential equations, Strasbourg, 1985.

Takabatake-cho 184-632

Nara 630, Japan 